\title{
Caracterização do grau tecnológico dos sericicultores no território Paraná Centro
}

\author{
Technological degree characterization of silkworm producers in the Paraná territory Center
}

\author{
Letícia Trindade Ataíde ${ }^{I^{*}}$ Ruy Seiji YamaokaII Maria Elizabeth da Costa Vasconcellos ${ }^{\text {II }}$ \\ José Roberto Pinto de Souza
}

\section{RESUMO}

\begin{abstract}
O objetivo deste trabalho foi caracterizar o grau tecnológico dos sericicultores e inferir os tipos de intervenções a serem empregadas para aumentar a produtividade em 17 municipios do centro do Estado do Paraná, os quais apresentam o menor Índice de Desenvolvimento Humano (IDH) desse Estado. Os dados originais de produção dos casulos verdes da safra 2004/2005 foram disponibilizados pela empresa de Fiação de Seda Bratac S.A. e submetidos à análise estatística multivariada de Componentes Principais. Esta técnica permitiu escolher quatro autovalores das 21 variáveis originais e assim utilizar o novo conjunto formado para realizar a análise descritiva dos dados. As quatro componentes selecionadas foram Capacidade/Intensidade de Produção, Qualidade de Produção, Eficiência na Utilização da Área e Retorno Financeiro da Propriedade. Devido ao grande volume de informações, realizou-se uma análise Cluster para as componentes dos 565 produtores, da qual resultou a formação de quatro grupos de características semelhantes. As análises dos grupos de produtores possibilitaram a observação de dois contrastes principais. Uma parte dos grupos apresenta produtores com maior capacidade e qualidade de produção de casulos na propriedade, e os demais compreendem produtores que exploram sua área produtiva de forma ineficiente.
\end{abstract}

Palavras-chave: Morus alba, Bombyx mori, indice de desenvolvimento humano (IDH), tecnologia de produção, agricultura familiar.

\section{ABSTRACT}

The goal of this research was to characterize the technological degree of silkworm producers and to infer the types of interventions to be employed to increase productivity in 17 localities in the central region of Paraná State, Southern Brazil, which has the lowest Human Development Index (HDI). The original data of 2004/2005 season from green cocoons production taken from the company Fiação de Seda Bratac S.A. and submitted to multivariate statistical analysis of Principal Components. This technique allowed to choose four eigenvalues of 21 original variables and thus to use the new set to perform a descriptive analysis of the data. The four components selected were capacity/intensity of production, quality of production, efficiency in the use of the area and financial return of the property. Due to the large volume of information, Cluster analysis was performed for the components of the 565 producers, resulting in four groups of similar characteristics. The analysis of group of producers enabled the observation of two main contrasts. One part of groups presented producers with greater capacity and quality of cocoon production in the property, and other include producers who exploit their productive area inefficiently.

Key words: Morus alba, Bombyx mori, human development index (HDI), technology of production, family farming.

\section{INTRODUÇÃO}

O Ministério do Desenvolvimento Agrário (MDA), com base no Índice de Desenvolvimento Humano (IDH), delimitou cinco Territórios Rurais no Estado do Paraná: Cantuquiriguaçu, Centro Sul, Paraná Centro, Sudoeste Paranaense e Vale do Ribeira (MDA/ SIT, 2002).

'Programa de Pós-graduação em Agronomia, Departamento de Agronomia, Universidade Estadual de Londrina (UEL), Rod. Celso Garcia Cid (PR 445), km 380, CP 6001, 86051-990, Londrina, PR, Brasil. E-mail: leticia_trindade@yahoo.com.br. *Autor para correspondência.

"Área de Fitotecnia, Instituto Agronômico do Paraná (IAPAR), Londrina, PR, Brasil.

IIIÁrea de Biometria, IAPAR, Londrina, PR, Brasil. 
O Território Paraná Centro, delimitado pelo MDA, engloba parte de três regionais definidas pela Secretaria da Agricultura e do Abastecimento do Estado do Paraná (SEAB/DERAL, 1998): Ivaiporã, Campo Mourão e Guarapuava. Esse território abrange 17 municípios: Altamira do Paraná, Boa Ventura de São Roque, Campina do Simão, Cândido de Abreu, Iretama, Laranjal, Manoel Ribas, Mato Rico, Nova Cantu, Nova Tebas, Palmital, Pitanga, Rio Branco do Ivaí, Roncador, Rosário do Ivaí, Santa Maria do Oeste e Turvo (MDA/ SIT, 2002). Essa região, localizada no centro do Paraná, apresenta o menor Índice de Desenvolvimento Humano (IDH) do Estado devido às precárias condições de vida da população, como também a diversos problemas ambientais relacionados às contaminações do solo e da água, entre outros (IPARDES, 2000; CENCI et al., 2005).

Os estudos da Cadeia Produtiva da Seda realizados nos anos de 1996 a 1998, no Paraná, revelaram uma evolução da sericicultura no Estado. A atividade é desenvolvida essencialmente por pequenos e médios agricultores que utilizam mão de obra familiar (WATANABE et al., 2000). A atividade sericícola tem a característica de proporcionar um grande número de empregos diretos e indiretos: aproximadamente 13,9 mil empregos diretos no campo e mais 2,0 mil nas indústrias. Gera, ainda, em torno de 3,0 mil empregos indiretos no setor de serviços (ZANETTI, 2007).

É estimado que $60 \%$ da rentabilidade da sericicultura dependem da produção de folhas por unidade de área e da qualidade de folhas de amoreira, e o desempenho das indústrias de seda refletirá no custo de produção das folhas de amora, dos casulos de seda, na produção de seda e no retorno aos sericicultores (PATIL et al., 2003; SANNAPPA et al., 2003).

As tecnologias usadas na atividade no Estado do Paraná são, em sua grande maioria, adaptadas, baseando-se nas empregadas no Estado de São Paulo, e/ou importadas dos principais países produtores de casulos de seda. Essas tecnologias descritas nos manuais, informes e outras publicações de alcance geral referem-se aos novos cultivares de amoreira, à calagem e adubação da cultura com base na extração de nutrientes e análise do solo e aos equipamentos para colheita e poda. São exemplos de tecnologias disponíveis desenvolvidas ou adaptadas para as condições do Paraná que ainda não estão disponíveis para uma grande parte dos sericicultores (WATANABE et al., 2000).

Os sistemas de exploração do bicho-da-seda no Estado do Paraná possuem grande diversidade de tecnologia e de estrutura de produção, diferenciandose principalmente em relação ao tipo de tração utilizada para a condução do amoreiral, tração animal, moto mecanizada e/ou a combinação deles, bem como o tipo de mão de obra. Na região central do Paraná, predomina a agricultura familiar.

O objetivo do presente trabalho foi caracterizar o grau tecnológico dos sericicultores e inferir os tipos de intervenções a serem feitas para aumentar a produção da atividade no Território Paraná Centro.

\section{MATERIAL E MÉTODOS}

A Fiação de Seda Bratac S.A. é a única empresa do setor da seda a atuar no Território Paraná Centro. A empresa disponibilizou os dados originais de 565 sericicultores da safra 2004/2005, envolvendo 17 municípios do Estado para a realização do presente trabalho.

Os dados fornecidos foram submetidos à análise estatística multivariada denominada de Análise de Componentes Principais (ACP). Foi obtida uma matriz (M) de dados com os 565 produtores sericícolas do Território Paraná Centro e 21 variáveis relacionadas à produção. A descrição das 21 variáveis originais relacionadas à produção sericícola dos agricultores utilizadas no trabalho são apresentadas na tabela 1 .

Os 17 municípios foram distribuídos em ordem alfabética e seus respectivos produtores foram também organizados por ordem alfabética dentro de cada município, atribuindo-se um número em ordem crescente de identificação para cada um dos 565 produtores utilizados na análise. As análises estatísticas foram realizadas no software SAS (1989a, b).

Da matriz M, obteve-se a matriz de correlações entre as 21 variáveis, de onde foram extraídas as raízes características, chamadas de autovalores, obtidas pelo método das componentes principais. Os quatro primeiros autovalores foram escolhidos pela capacidade de explicação de $75 \%$ da variação total, possibilitando resumir a informação dos dados originais nessas novas variáveis.

A escolha de quatro autovalores foi relacionada com o total da variância acumulada de cada autovalor, pois se acreditava que a contribuição de $75 \%$ pudesse substituir as variáveis originais sem muita perda de informação, desde que tivesse uma boa interpretação prática do problema (REGAZZI, 2000).

\section{RESULTADOS E DISCUSSÃO}

Os 565 sericicultores do Território Paraná Centro apresentaram níveis de produtividade com elevada heterogeneidade, possivelmente em virtude da 
Tabela 1 - Descrição das variáveis quanto à produção sericícola dos agricultores do Paraná Centro utilizadas para análise estatística multivariada de Componentes Principais.

\begin{tabular}{|c|c|}
\hline Variável & Descrição \\
\hline V1 & Área total da propriedade (ha) \\
\hline $\mathrm{V} 2$ & Área total do amoreiral implantado (ha) \\
\hline V3 & Área total da cama de criação do bicho-da-seda $\left(\mathrm{m}^{2}\right)$ \\
\hline V4 & Área total da sirgaria de criação do bicho-da-seda $\left(\mathrm{m}^{2}\right)$ \\
\hline V5 & Capacidade produtiva total da sirgaria em número de caixas de bicho-da-seda \\
\hline V6 & Capacidade produtiva utilizada pelo produtor em número de caixas de bicho-da-seda \\
\hline V7 & Quantidade de casulos de $1^{\mathrm{a}} \mathrm{A}\left(1^{\mathrm{a}}\right.$ qualidade) por caixa $(\mathrm{kg})$ \\
\hline V8 & Peso de um casulo $(\mathrm{g})$ \\
\hline V9 & Número de casulos em $500 \mathrm{~g}$ \\
\hline V10 & Teor de seleção (\%) \\
\hline V11 & Taxa de Retorno de casulos \\
\hline V12 & Teor de seda $(\%)$ \\
\hline V13 & Peso total de casulos (sem anafaia) \\
\hline V14 & Valor total de casulos ( $\mathrm{R} \$$ com anafaia) \\
\hline V15 & Peso total de casulos de $1^{\mathrm{a}} \mathrm{A}(\mathrm{kg})$ \\
\hline V16 & Valor total de casulos de $1^{\mathrm{a}} \mathrm{A}$ ( $\mathrm{R} \$$ sem prêmio) \\
\hline V17 & Valor total do prêmio $(\mathrm{R} \$)$ \\
\hline V18 & Valor total de casulos de $1^{\mathrm{a}} \mathrm{A}$ ( $\mathrm{R} \$$ com prêmio $)$ \\
\hline V19 & Valor por quilo de casulos de $1^{\mathrm{a}} \mathrm{A}$ ( $\mathrm{R} \$$ com prêmio) \\
\hline V20 & Duração da safra (dias) \\
\hline V21 & Produtividade (quilos de casulos de $1^{\mathrm{a}} \mathrm{A}$ por hectare de amora $-\mathrm{kg} 1^{\mathrm{a}} \mathrm{A} \mathrm{ha}^{-1}$ ) \\
\hline
\end{tabular}

diversidade tecnológica, que deve ser identificada pontualmente, e assim permitir uma intervenção no sistema de produção, e ainda de informações tecnológicas, que não os têm atingido de forma satisfatória.

A somatória desses fatores, que vão desde a condução do amoreiral no campo até a alimentação das lagartas, interfere diretamente nas variáveis de produção, podendo favorecer os agricultores a alcançarem níveis produtivos mais elevados do que os obtidos atualmente, além de uma produtividade mais homogênea.

Como se pode ver na tabela 2, a análise das informações geradas a partir dos dados indica que a primeira componente principal (Y1), composta pelas variáveis que envolvem a área, a capacidade de produção, além da quantidade e do peso do casulo, explica $42,88 \%$ da variação total e abrange a maioria das características observáveis nas variáveis originais. A segunda componente (Y2), quantidade, peso e preço de casulos, explica $16,93 \%$ da variação total. A terceira componente (Y3), área de criação das lagartas e sua utilização, contribui com $8,16 \%$. Por fim, a quarta componente (Y4), duração da safra e produtividade, contribui com $7,02 \%$ da variação total. Assim, pode-se dizer que essas quatro componentes ( $\mathrm{Y} 1, \mathrm{Y} 2, \mathrm{Y} 3 \mathrm{e}$ Y 4 ) substituem as 21 variáveis originais sem perda de informação, sendo responsáveis por $75 \%$ da variação total.
Essas quatro componentes (Yi) forneceram informações suficientes para associar as variáveis originais ao grau tecnológico presente nas propriedades sericícolas. De acordo com MINGOTTI (2005) e SILVA \& PADOVANI (2006), quanto maior for a retenção da variação total em um número menor de combinações lineares, melhor será a aplicação prática desse procedimento aos dados experimentais.

As quatro componentes principais ( $\mathrm{Y} 1, \mathrm{Y} 2$, Y3 e Y4) e seus respectivos autovetores, que são os coeficientes ou pesos de cada variável na equação que irá representar cada componente Yi, estão listados na tabela 2. Entende-se, então, que para a componente $\mathrm{Y} 1$, as variáveis V14 e V18, relacionadas com o valor total de casulos, são determinantes, por contribuírem com autovetores de maiores coeficientes, 0,3226 e 0,3227 , respectivamente. As variáveis relacionadas com o peso do casulo (V13 e V15), com os valores do casulo sem prêmio (V16) e com prêmio (V17) e com a capacidade produtiva utilizada (V6) contribuíram com os autovetores de coeficientes 0,$3107 ; 0,3126 ; 0,3174$; 0,3020 e 0,2775 , respectivamente. O mesmo raciocínio foi seguido para as variáveis das demais componentes.

As variáveis originais e as componentes principais foram então correlacionadas entre si (Tabela $3)$. Verificou-se que, das 21 variáveis originais, as que mais se correlacionaram com a primeira componente principal (Y1) foram as referentes ao peso e valor dos 
Tabela 2 - Autovetores e Autovalores da Matriz de Correlação entre as variáveis originais e as componentes principais.

\begin{tabular}{lllll}
\hline Variável & Y1 & Y2 & Y3 & Y4 \\
\hline V1 & 0,0600 & 0,1066 & 0,2370 & 0,0626 \\
V2 & 0,1393 & 0,2240 & 0,2128 & 0,0957 \\
V3 & 0,1950 & 0,2945 & 0,3586 & 0,0991 \\
V4 & 0,1769 & 0,2929 & 0,3597 & 0,0784 \\
V5 & 0,1972 & 0,2846 & 0,3455 & $-0,0973$ \\
V6 & 0,2775 & 0,2125 & 0,2454 \\
V7 & 0,1749 & $-0,3221$ & $-0,1716$ \\
V8 & 0,1524 & $-0,3546$ & 0,1790 \\
V9 & $-0,1553$ & 0,3558 & $-0,02292$ & $-0,1882$ \\
V10 & $-0,0920$ & 0,1962 & 0,4860 \\
V11 & 0,1001 & $-0,1563$ & $-0,2549$ & 0,2179 \\
V12 & $-0,2115$ & $-0,1436$ & $-0,0193$ \\
V13 & 0,1532 & $-0,2312$ & $-0,0056$ \\
V14 & 0,0968 & 0,1224 & $-0,0257$ \\
V15 & 0,3107 & $-0,2220$ & $-0,0030$ \\
V16 & 0,3226 & $-0,1694$ & $-0,0295$ \\
V17 & 0,3126 & $-0,2153$ & $-0,0076$ \\
V18 & 0,3174 & $-0,1990$ & 0,1215 \\
V19 & 0,3020 & 0,0914 & $-0,5544$ \\
V20 & 0,3227 & 0,0671 & $-0,4385$ \\
V21 & 0,1930 & $-0,1525$ & $-0,1682$ & 1,4750 \\
Autovalor & 0,0702 & 0,0316 & 0,2165 & 7,02 \\
\% explicada & 0,1865 & $-0,3415$ & 0,1090 & $-0,0638$ \\
\hline
\end{tabular}

casulos (V13 a V18), com correlação maior que 0,9, indicando que essas variáveis estão representadas no primeiro eixo da componente principal. Assim, Y1 apresenta correlação alta e positiva com V13 a V18, e a capacidade produtiva utilizada (V6) tem correlação média e positiva com área e capacidade produtiva da sirgaria (V3 a V5), preço por quilo de casulos (V19) e produtividade (V21).

Essas variáveis estão relacionadas com a capacidade de produção da propriedade, podendo-se então, denominar a primeira componente (Y1) de Capacidade e Intensidade de Produção. Logo, Y1 representa propriedades com maiores valores relacionados à área e à capacidade de criação, ao peso e valor dos casulos e à produtividade (V3, V5, V6, V7, V8, V14, V16, V17, V18, V19e V21).

A segunda componente principal (Y2) pode ser denominada de Qualidade Produtiva da Propriedade, tendo sida descrita de acordo com as propriedades que possuem maiores área e capacidade produtiva (V3, V4, V5 e V9). Porém, essas propriedades apresentam também menor quantidade e peso de casulos (V7 e V8) $\mathrm{e}$, consequentemente, menor valor por quilo de casulos (V19).

A terceira componente principal (Y3) foi denominada de Eficiência na Utilização da Área, sendo representada pelas propriedades com maiores área total da cama de criação do bicho-da-seda e área total da sirgaria (V3 e V4).

A quarta componente principal (Y4) foi chamada de Retorno Financeiro da Propriedade e representa as propriedades que detêm o maior retorno de casulos (V11), as menores duração da safra (V20) e as maiores produtividades (V21).

Para identificar e classificar cada produtor por essas quatro componentes principais, construiuse uma nova matriz de $4 \times 565$ com os valores dos escores para cada produtor. Para a caracterização do grau tecnológico dos produtores, efetuou-se uma análise Cluster (análise de agrupamento) sobre os valores dos escores para as 565 propriedades pelo método da ligação pela média, de tal forma que se pode agrupar os produtores em quatro grupos, de acordo com as características semelhantes das propriedades.

O diagnóstico dos produtores em cada grupo identificou as propriedades pelo seu maior ou menor uso de tecnologias, tais como a utilização de cultivares de amoreira adaptadas às condições locais e ausência de mistura varietal; adoção de calagem e adubação baseadas na análise do solo e na extração de nutrientes pelas plantas; e melhoria no sistema de colheita de ramas de amoreira e na realização das podas (WATANABE et al., 2000). Observou-se que há diversificação dos grupos devido à grande variabilidade 
Tabela 3 - Correlação entre as variáveis originais e as componentes principais (Yi).

\begin{tabular}{lllll}
\hline Variáveis & Y1 & Y2 & Y3 & Y4 \\
\hline V1 & 0,1800 & 0,2010 & 0,3102 & 0,0761 \\
V2 & 0,4182 & 0,4224 & 0,2785 & 0,1163 \\
V3 & 0,5854 & 0,5553 & 0,4694 & 0,1203 \\
V4 & 0,5309 & 0,5522 & 0,0952 \\
V5 & 0,5918 & 0,5367 & $-0,1182$ \\
V6 & 0,8329 & 0,4006 & 0,4522 & 0,2981 \\
V7 & 0,5248 & $-0,6073$ & $-0,28855$ \\
V8 & 0,4573 & $-0,6686$ & $-0,0301$ & 0,2174 \\
V9 & $-0,4662$ & 0,6708 & $-0,2286$ \\
V10 & 0,3700 & 0,3276 & 0,5903 \\
V11 & $-0,2763$ & $-0,2947$ & 0,2647 \\
V12 & $-0,3988$ & $-0,3336$ & $-0,0235$ \\
V13 & 0,3006 & $-0,3026$ & $-0,0068$ \\
V14 & 0,4597 & 0,1602 & $-0,0313$ \\
V15 & 0,9326 & 0,0614 & $-0,0037$ \\
V16 & 0,9682 & 0,1723 & $-0,2906$ & $-0,0359$ \\
V17 & 0,9382 & $-0,2217$ & $-0,0092$ \\
V18 & 0,9527 & $-0,2605$ & 0,1476 \\
V19 & 0,9064 & $-0,2875$ & $-0,6733$ \\
V20 & 0,9686 & 0,0597 & $-0,5326$ \\
V21 & 0,5794 & $-0,6440$ & $-0,0167$ & \\
\hline
\end{tabular}

dos criadores. As caracterizações dos produtores nos grupos são apresentadas a seguir:

Grupo 1 (76 produtores): apresentam alta capacidade produtiva (V5), baixo peso de casulo (V8) e maior número de casulos em $500 \mathrm{~g}$ (V9). A maioria dos criadores possui baixa Qualidade Produtiva da Propriedade(Y2). Além disso, possuem baixa Eficiência na Utilização da Área (Y3), o que influencia o Retorno Financeiro da Propriedade (Y4), gerando um lucro insatisfatório.

Grupo 2 (142 produtores): apresentam capacidade de produção baixíssima, mesmo com alguns valores positivos para Qualidade Produtiva da Propriedade (Y2). Isso é devido à ineficiente utilização da capacidade produtiva (V5), explicado pela Eficiência na Utilização da Área (Y3), o que reflete diretamente em Retorno Financeiro da Propriedade (Y4).

Grupo 3 (345 produtores): formado pela maioria dos produtores, que possuem Capacidade e Intensidade de Produção (Y1) e Qualidade Produtiva da Propriedade (Y2) relativamente baixas. Isso é devido à ineficiente Utilização da Área (Y3), do mesmo modo que o ocorrido com os produtores do grupo 2. Com o Retorno Financeiro da Propriedade (Y4), observou-se que a maioria deles apresentava pequenos prejuízos ou poucos lucros.

Grupo 4 ( 2 criadores): apresentaram as mais altas Capacidade e Intensidade de Produção (Y1), o que pode ser explicado pela melhor utilização da área (Y3). Além disso, a atividade é conduzida com maior número de caixas de lagartas por criada, embora essas duas propriedades ainda não estejam em sua capacidade máxima de utilização. Apesar da elevada produtividade deste grupo, ocorreu um retorno de lagartas aquém do desejado.

Ao se comparar os quatro grupos, verificouse que as maiores correlações estão ligadas ao grupo 4 , que têm os melhores produtores, seguidos dos grupos 1,3 e 2, com os criadores de produções mais baixas, destacando-se o grupo 2 por apresentar a menor média dentre os demais para todas as variáveis analisadas.

Os criadores inseridos no grupo 3 apresentaram dados médios baixos em relação aos criadores dos grupos 1 e 4, exceto para área de criação das lagartas (V4) e peso de casulo (V8), o que demonstra melhor aproveitamento do espaço utilizado. Isso interferirá positivamente na quantidade de casulos por caixa (V7) e, consequentemente, no aumento da produção. Portanto, esse grupo possui boas chances de se atingir um patamar mais elevado de produção, equiparando-se ao grupo 1 e, até mesmo, ao grupo 4 , se feita a capacitação tecnológica dos produtores através de profissionais devidamente treinados.

Comparando-se os dados de produtividade entre os grupos, verificou-se que o grupo 2 apresentou

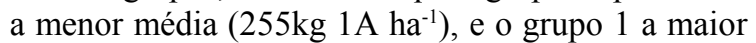

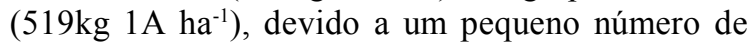
produtores com alta produtividade, amoreirais novos e com variedades mais produtivas (CENCI et al., 2005). 
Os produtores inseridos no grupo 3, apesar de apresentarem média de produtividade de $376 \mathrm{~kg} 1 \mathrm{~A}$

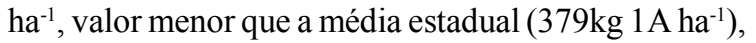
possuem sistemas de exploração passíveis de serem incrementados tecnologicamente, como manejo adequado do solo e adubação. Esses fatores influenciam significativamente a produtividade e a qualidade do casulo por unidade de área, proporcionando melhoria na produtividade (WATANABE et al., 2000).

Com o emprego das tecnologias atualmente existentes no Paraná, alguns criadores têm atingido produções acima de $1000 \mathrm{~kg} \mathrm{ha}^{-1}$ ano $^{-1}$. Esse valor pode ser alcançado com o emprego de cultivares de amoreira selecionadas de acordo com as condições edafoclimáticas do local de plantio, com a adoção de manejos adequados de condução da atividade no que se refere à adubação, com a colheita dos ramos de amoreira para alimentação das lagartas por meio de sistema mecanizado de forma adequada para que não abale em demasia a touceira da planta (WATANABE et al., 2000).

A transferência de técnicas mais modernas aos produtores proporcionará melhoria na qualidade $\mathrm{e}$ quantidade da produção de folhas de amoreira, como também na economia de nutrientes com a realização da calagem e adubação das plantas restritas a sua necessidade nutricional. Além disso, essa transferência poderá incrementar o número de brotações e reduzir os danos às touceiras das plantas. Desse modo, a sericicultura poderá ser uma aliada do produtor rural para um incremento quali-quantitativo na atividade, refletindo-se em retorno financeiro e social.

\section{CONCLUSÃO}

Há grandes diferenças no processo de produção sericícola entre os produtores do Território Paraná Centro. O conhecimento das diferenciações que ocorrem entre os produtores é fundamental para o desenvolvimento do setor e o correto direcionamento das ações de políticas públicas.

Os quatro grupos de produtores se distinguem por dois contrastes principais. Os grupos 4 e 1 apresentam produtores com maior capacidade e qualidade de produção de casulos na propriedade, e os grupos 3 e 2 compreendem produtores que exploram sua área produtiva de forma ineficiente.

\section{AGRADECIMENTOS}

Ao Ministério do Desenvolvimento Agrário (MDA), e à Fundação de Apoio à Pesquisa e ao Desenvolvimento do Agronegócio (FAPEAGRO) pelo apoio financeiro, à Fiação de Seda Bratac S.A. pelos dados e à Fundação Araucária pela bolsa de mestrado ao primeiro autor.

\section{REFERÊNCIAS}

CENCI, D.R. et al. Plano de ações - Paraná centro. Ijuí: Unijuí, 2005. 91p.

IPARDES - Instituto Paranaense de Desenvolvimento Econômico e Social. Índice de desenvolvimento humano municipal segundo as unidades de federação - Brasil 1991/2000. 2000. Acesso em: 30 mar. 2007. Online. Disponível em: <http://www.ipardes.gov.br/>.

MDA - Ministério do Desenvolvimento Agrário / SIT - Sistema de Informações Territoriais. Mapas de Territórios Rurais, 2002. Acesso em: 30 mar. 2007. Online. Disponível em: $<$ http://serv-sdt-1.mda.gov.br/sit/banco_mapa.php>.

MINGOTTI, S.A. Análise de dados através de métodos de estatística multivariada: uma abordagem aplicada. Belo Horizonte: UFMG, 2005. 297p.

PATIL, S.V. et al. Evaluation of improved mulberry genotypes based on leaf yield, quality and bioassay using silkworm race, PM X NB4D2. Indian Journal of Sericulture, India, v.42, n.1, p.61-62, 2003.

REGAZZI, A.J. Análise multivariada, notas de aula. Viçosa: Departamento de Informática da Universidade de Viçosa, 2000. V.2. (INF 766).

SANNAPPA, B. et al. Economic evaluation of feeding methods and regimes in silkworm, Bombyx mori. Journal of Ecobiology, India, v.15, n.6, p.401-406, 2003.

SAS - Institute Inc. SAS/STAT User's guide. Version 6, 4.ed Carey, NC, 1989a. V.1, 943p.

SAS - Institute Inc. SAS/STAT User's guide. Version 6, 4.ed. Carey, NC, 1989b. V.2, 843p.

SEAB - Secretaria da Agricultura e do Abastecimento do Paraná / DERAL - Departamento de Economia Rural. Núcleos Regionais, 1998. Acesso em: 29 mar. 2007. Online. Disponível em: $<\mathrm{http} / /$ / www.seab.pr.gov.br/modules/conteudo/ conteudo.php?conteudo $=73>$.

SILVA, N.R. da; PADOVANI, C.R. Utilização de componentes principais em experimentação agronômica. Energia na Agricultura, Botucatu, v.21, n.4, p.98-113, 2006. Disponível em: <http://www.fca.unesp.br/CD REVISTA ENERGIA vol8/ vol2 1 n42006/ artigos/Nilza\%20Regina\%20- da\%20Silva.pdf $>$. Acesso em: 20 maio, 2007.

WATANABE, J.K. et al. Cadeia produtiva da seda: diagnósticos e demandas atuais. Londrina: IAPAR, 2000. $129 \mathrm{p}$.

ZANETTI, R. Introdução à sericicultura. Notas de aula. DEN/UFLA, Lavras, 18 maio, 2007. Especiais. Acesso em: 18 maio, 2007. Online. Disponível em: <http://www.den.ufla.br/ Professores/Ronald/Disciplinas/Notas\%20 Aula/ Sericicultura\%20introducao.pdf $>$. 